\title{
Avaliação epidemiológica da ansiedade dos pacientes ao tratamento odontológico
}

\author{
Epidemiological evaluation of patients' anxiety to dental treatment \\ Valoración epidemiológica de la ansiedad de los pacientes ante el tratamiento odontológico
}

Recebido: 06/11/2021 | Revisado: 15/11/2021 | Aceito: 17/11/2021 | Publicado: 20/11/2021

\author{
Lorena Fernandes Soares \\ ORCID: https://orcid.org/0000-0002-2217-2458 \\ Faculdade Independente do Nordeste, Brasil \\ E-mail: lorenafs05@hotmail.com \\ Rubiane Farias Alves Pessoa \\ ORCID: https://orcid.org/0000-0001-6661-1857 \\ Faculdade Independente do Nordeste, Brasil \\ E-mail: rubianefarias@hotmail.com \\ Karina Sarno Paes Alves Dias \\ ORCID: https://orcid.org/0000-0003-4840-2335 \\ Faculdade Independente do Nordeste, Brasil \\ E-mail: karinasarnopad@gmail.com
}

\begin{abstract}
Resumo
Objetivo: Avaliar o nível de ansiedade odontológica em pacientes de uma clínica-escola no sudoeste da Bahia. Material e Métodos: Foram abordados 80 pacientes sob atendimento odontológico para este estudo, após o consentimento em assinar o Termo de Consentimento Livre e Esclarecido, com coleta de dados realizada através de dois questionários, um socioeconômico e outro de avaliação de ansiedade. Todos os protocolos de segurança descritos pela atual situação pandêmica e de saúde foram cumpridos rigorosamente. Os dados coletados foram tabulados em planilha Excel e transferidos para o programa estatístico SPSS (IBM) versão 25.0. Resultado: O estudo apresentou 77,5\% dos entrevistados do sexo feminino, $46,3 \%$ com renda de até 1 salário mínimo e $36,1 \%$ completaram o $2^{\circ}$ grau. $31,2 \%$ dos pacientes só visitaram o dentista quando sentiram dor, 28,7\% se sentiram incomodados com a anestesia, e $49 \%$ apresentaram ansiedade nível leve. Conclusão: A ansiedade se mostrou presente entre os pacientes, e a maneira de amenizar essa situação é através da odontologia humanizada, lidando com o paciente de maneira direcionada e específica.
\end{abstract}

Palavras-chave: Ansiedade ao tratamento odontológico; Escala de ansiedade frente a teste; Saúde bucal; Assistência odontológica.

\begin{abstract}
Objective: To evaluate the level of dental anxiety in patients at a teaching clinic in southwestern Bahia. Material and Methods: 80 patients were approached under dental care for this study, after consent to sign the Informed Consent, with data collection performed through two questionnaires, one socioeconomic and one of anxiety assessment. All security protocols described by the current pandemic and health situation were met strictly. The collected data were tabulated in excel spreadsheet and transferred to statistical program SPSS (IBM) version 25.0. Result: The study presented $77.5 \%$ of female interviewed, $46.3 \%$ with income up to 1 basic salary and $36.1 \%$ completed high school. $31.2 \%$ of patients only visited the dentist when they felt pain, $28.7 \%$ felt uncomfortable with the anesthesia and $49 \%$ had mild anxiety. Conclusion: Anxiety is present among patients, and a way to ease this situation is through humanized dentistry, dealing with the patient in a direct and specific way.
\end{abstract}

Keywords: Dental anxiety; Test anxiety scale; Oral health; Dental care.

\section{Resumen}

Objetivo: Evaluar el nivel de ansiedad dental en pacientes de una clínica docente del suroeste de Bahía. Material y Métodos: Se abordó a 80 pacientes sometidos a atención odontológica para este estudio, previo consentimiento para firmar el Formulario de Consentimiento Informado, con recolección de datos realizada a través de dos cuestionarios, uno socioeconómico y otro para evaluación de ansiedad. Todos los protocolos de seguridad descritos por la pandemia actual y la situación de salud se cumplieron estrictamente. Los datos recolectados se tabularon en una hoja de cálculo de Excel y se transfirieron al programa estadístico SPSS (IBM) versión 25.0. Resultado: El estudio mostró que el 77,5\% de los encuestados eran mujeres, el 46,3\% con ingresos de hasta 1 salario mínimo y el 36,1\% completó la escuela secundaria. El 31,2\% de los pacientes solo visitaron al dentista cuando sintieron dolor, el 28,7\% se sintió incómodo con la anestesia y el $49 \%$ tenía ansiedad leve. Conclusión: la ansiedad estuvo presente en los pacientes, y la forma de paliar esta situación es a través de la odontología humanizada, tratando al paciente de manera focalizada y específica. 
Palabras clave: Ansiedad al tratamiento odontológico; Escala de ansiedad ante pruebas; Salud bucal; Atención odontológica.

\section{Introdução}

O medo e a ansiedade são sentimentos naturais dos seres humanos, mas podem atrapalhar a partir do momento em que interferem no próprio cotidiano de forma negativa (Silva, 2020). No âmbito odontológico, esses sentimentos podem ser classificados como objetivos, quando a experiência já foi vivenciada durante um tratamento odontológico anterior, e subjetivos, quando uma experiência negativa foi relatada por outra pessoa acerca de um procedimento de saúde (Peronio, Silva, \& Dias, 2018).

Os pacientes com ansiedade odontológica se dividem em quatro grupos: os que têm ansiedade pelo procedimento em si, os que têm insegurança em relação ao dentista, os que apresentam ansiedade generalizada e os que temem as emergências médicas dentro do consultório (Maulina, Djustiana, \& Shahib, 2017). Essas situações podem gerar fisiologicamente sudorese, tremores, aumento da frequência cardíaca e respiratória, e até desmaios. Já no âmbito psicológico, ocasionam apreensão, malestar, angústia, insegurança e dificuldades de concentração e interação social (Appukuttan, 2016).

A ansiedade dentária está relacionada com o medo da dor, e intimamente ligada com a sensação de impotência que o paciente sente na cadeira odontológica, por não possuir o controle da situação (Appukuttan, 2016). Diante disso, o medo pode ser originário do motor de alta rotação, das brocas dentárias, da agulha de anestesia, do procedimento cirúrgico de exodontia, dentre outros (Peronio et al., 2018). A aversão irracional à odontologia é denominada “odontofobia”, e foi definida pelo Manual de Diagnóstico e Estatística de Transtornos Mentais e pela Classificação Estatística Internacional de Doenças e Problemas Relacionados à Saúde (Appukuttan, 2016).

A manutenção da saúde bucal é um fator agravante para os odontofóbicos, pois os mesmos tendem a protelar os procedimentos dentários, comparecendo ao consultório somente quando há uma urgência, e essa situação pode se tornar irreversível e possivelmente mutiladora (Peronio et al., 2018). Esse tipo de comportamento ocasiona uma saúde bucal precária, com problemas periodontais, perdas dentárias e elevado índice de cárie (Appukuttan, 2016). Muitas vezes, o paciente passa a administrar fármacos como analgésicos e anti-inflamatórios para amenizar a dor, o que agrava ainda mais o seu caso clínico (Stefano, 2019).

O termo apropriado para esse comportamento é denominado Ciclo vicioso de medo dental ou Ciclo de evitação dentária, em que o paciente fica ansioso para o tratamento, pois tem medo de sentir dor e adia sua visita ao dentista. Esse retardo em procurar o atendimento gera diversos problemas dentários, muitas vezes, com necessidade de tratamento de urgência, que pode ser traumático e invasivo, reforçando, ainda mais, o seu medo e levando-o ao declínio de uma futura consulta. Dessa forma, o paciente entra em um espiral autodestrutivo (Appukuttan, 2016; Stefano, 2019).

Assim, considerando o impacto negativo que a ansiedade pode exercer sobre o tratamento odontológico, o objetivo do presente estudo foi avaliar a prevalência de ansiedade odontológica em pacientes atendidos em uma Faculdade de Odontologia do Sudoeste da Bahia no ano de 2021, considerando o que os deixou mais ansiosos e avaliando proporções de ansiedade entre os sexos, idades e nível de escolaridade.

\section{Metodologia}

Conforme fundamento metodológico de Pereira et al. (2018), esta pesquisa trata-se de um estudo quantitativo com abordagem observacional, descritivo, transversal que buscou avaliar, por meio de questionários, o nível de ansiedade odontológica dos pacientes atendidos na Clínica de Odontologia da Faculdade Independente do Nordeste (FAINOR), identificando o que os deixam mais ansiosos e as proporções dessa ansiedade entre os sexos, idades e nível de escolaridade. 
Foram selecionados 80 pacientes, os quais foram abordados, um de cada vez, na sala de espera pelo acadêmico responsável pela pesquisa e convidados a responder o questionário. Todos os protocolos de segurança descritos pela atual situação pandêmica do covid-19 e de saúde foram cumpridos rigorosamente.

O formulário de coleta de dados foi composto por duas partes, uma com questões de identificação pessoal e outra com questões específicas para estabelecer o grau de ansiedade dos pacientes ao tratamento odontológico, sendo utilizado a escala DAS (Dental Anxiety Scale) proposta inicialmente por Corah (1969) e traduzida por Pereira e Queluz (2000).

A aplicação do questionário foi executada antes da consulta agendada pelo acadêmico que prestou atendimento àquele participante da pesquisa, o que ocorreu em ambos os turnos de atendimento do centro odontológico (matutino e vespertino), obedecendo ao critério do horário de atendimento destes pacientes, de forma que não atrapalhou o fluxo de atendimento do Núcleo de Odontologia. Todos os participantes da pesquisa foram informados sobre o propósito do estudo e assinaram o Termo de Consentimento Livre e Esclarecido (TCLE), além de terem sido informados sobre a importância de guardar uma via de cada documento utilizado na pesquisa (Questionário e TCLE).

Foram incluídos pacientes da clínica escola, maiores de 18 anos, alfabetizados, de ambos os sexos, que concordaram em assinar o TCLE. Foram excluídas mulheres em período de gestação/lactação/menstruação, indivíduos menores de 18 anos, não alfabetizados, que não concordaram em responder os questionários e/ou assinar o TCLE, além de questionário preenchido incompletamente.

Os dados coletados através dos questionários foram tabulados e receberam tratamento estatístico descritivo (frequências, médias e medidas de dispersão) e analítico (Teste de Kruskal-Wallis e U de Mann-Whitney, ambos com significância fixa em 5\%) através do Statistical Package for the Social Sciences - SPSS (IBM Corporation, Armonk, EUA), verão 22.2 para Windows. As tabelas e gráficos foram plotados pelo Microsoft Excel (Microsoft, Redmond, EUA).

O presente estudo foi aprovado pelo Comitê de Ética e Pesquisa, segundo resolução vigente para Ética em Pesquisa em Seres Humanos n 466/12 do Conselho Nacional de Saúde (Ministério da Saúde, DF), além de autorização da Instituição para coleta de dados.

\section{Resultados}

A amostra composta por 80 indivíduos possui perfil predominantemente feminino 62 (77,5\%), com idade compreendida entre 45 e 54 anos 23 (28,8\%), renda de até 1 salário mínimo 37 (46,3\%) e de escolaridade $2^{\circ}$ grau completo 29 (36,1\%), conforme mostra a Tabela 1. 
Research, Society and Development, v. 10, n. 15, e347101522917, 2021

(CC BY 4.0) | ISSN 2525-3409 | DOI: http://dx.doi.org/10.33448/rsd-v10i15.22917

Tabela 1. Características biosociodemográficas da amostra.

\begin{tabular}{|c|c|c|}
\hline Variáveis & $\mathbf{n}$ & $\%$ \\
\hline \multicolumn{3}{|l|}{ Sexo } \\
\hline Masculino & 18 & 22,5 \\
\hline Feminino & 62 & 77,5 \\
\hline \multicolumn{3}{|l|}{ Faixa etária, anos } \\
\hline 15 a 24 & 7 & 8,8 \\
\hline 25 a 34 & 16 & 20,0 \\
\hline 35 a 44 & 20 & 25,0 \\
\hline 45 a 54 & 23 & 28,7 \\
\hline 55 a 64 & 10 & 12,5 \\
\hline$<65$ & 4 & 5,0 \\
\hline \multicolumn{3}{|l|}{ Renda, salários mínimos } \\
\hline Até 1 & 37 & 46,3 \\
\hline De 1 a 3 & 35 & 43,6 \\
\hline De 3 a 5 & 5 & 6,3 \\
\hline Mais que 5 & 3 & 3,8 \\
\hline \multicolumn{3}{|l|}{ Escolaridade } \\
\hline $1^{\circ}$ grau incompleto & 17 & 21,3 \\
\hline $1^{\circ}$ grau completo & 7 & 8,8 \\
\hline $2^{\circ}$ grau incompleto & 5 & 6,3 \\
\hline $2^{\circ}$ grau completo & 29 & 36,1 \\
\hline superior incompleto & 10 & 12,5 \\
\hline superior completo & 12 & 15,0 \\
\hline
\end{tabular}

Fonte: Dados da pesquisa.

De acordo com a Tabela 2, no que se refere aos dados odontológicos, a amostra afirmou realizar consulta majoritariamente somente quando sente dor 25 (31,2\%), bem como ser a procedência do incômodo a aplicação da anestesia em $23(28,7 \%)$ dos casos. 
Tabela 2. Características clínicas da amostra.

\begin{tabular}{lcc}
\hline Variáveis & n & \% \\
\hline Frequência de consulta & 22 & 27,5 \\
a cada 6 meses & 5 & 6,3 \\
não me lembro & 19 & 23,7 \\
1 vez por ano & 25 & 31,2 \\
somente quando tenho dor & 9 & 11,3 \\
1 vez cada 2 anos & & \\
Procedência do incômodo & 12 & 15,0 \\
Alta rotação & 28,7 \\
Anestesia & 23 & 21,3 \\
Cirurgias & 17 & 27,5 \\
Nenhum & 22 & 7,5 \\
Outros & 6 & \\
\hline
\end{tabular}

Fonte: Dados da pesquisa.

Quanto à identificação da ansiedade, dado pelo questionário Corah, a amostra apresentou predominância do nível leve em 39 (49\%) das situações. Gráfico 1.

Gráfico 1. Nível de ansiedade odontológica da amostra.

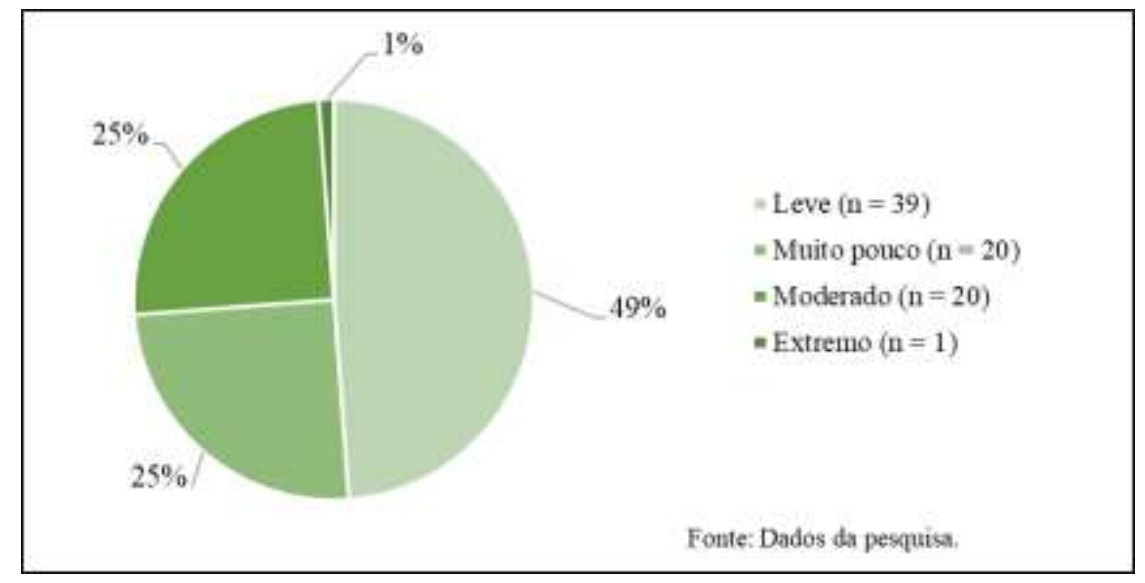

Fonte: Dados da pesquisa.

Com o auxílio do Gráfico 2, é possível inferir que não há diferença estatística significante entre o escore da escala de ansiedade odontológica e o sexo da amostra $(\mathrm{p}=0,736)$, levando em consideração que indivíduos do sexo masculino apresentam médias de $8,16( \pm 3,43)$, enquanto do sexo feminino $8,50( \pm 3,44)$. 
Research, Society and Development, v. 10, n. 15, e347101522917, 2021

(CC BY 4.0) | ISSN 2525-3409 | DOI: http://dx.doi.org/10.33448/rsd-v10i15.22917

Gráfico 2. Distribuição da escala de ansiedade odontológica pelo sexo da amostra.

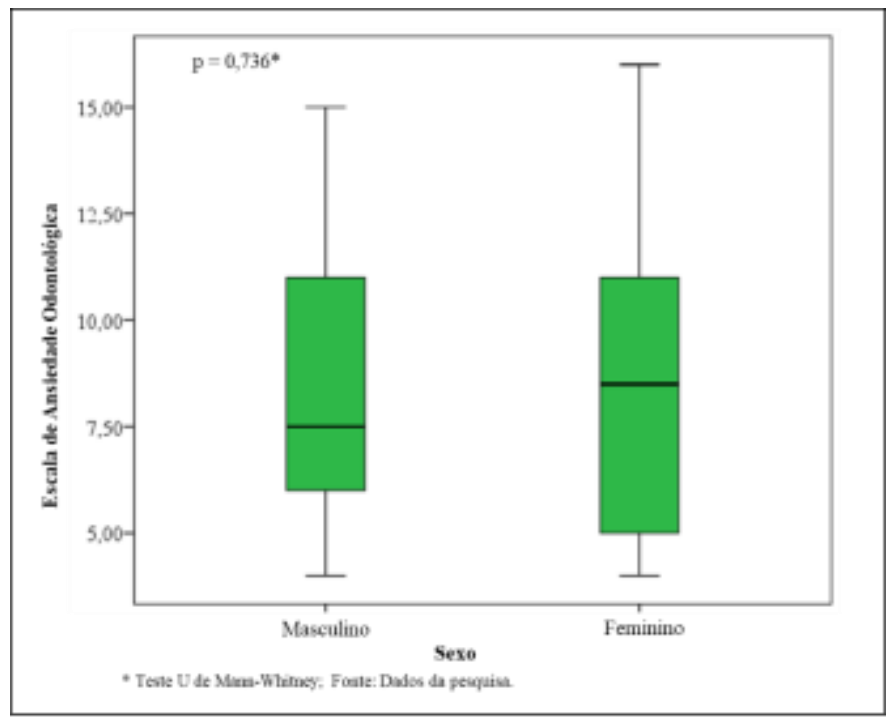

Fonte: Dados da pesquisa.

A amostra apresentou uma tendência decrescente do nível de ansiedade por faixa etária de forma estatisticamente significante $(\mathrm{p}=0,019)$, uma vez que pessoas de 15 a 24 anos possuíam média 10,42 $( \pm 2,87)$, enquanto maiores que 65 anos média de 7,0 ( $\pm 2,58)$ em nível de ansiedade. Gráfico 3.

Gráfico 3. Distribuição da escala de ansiedade odontológica pela faixa etária da amostra.

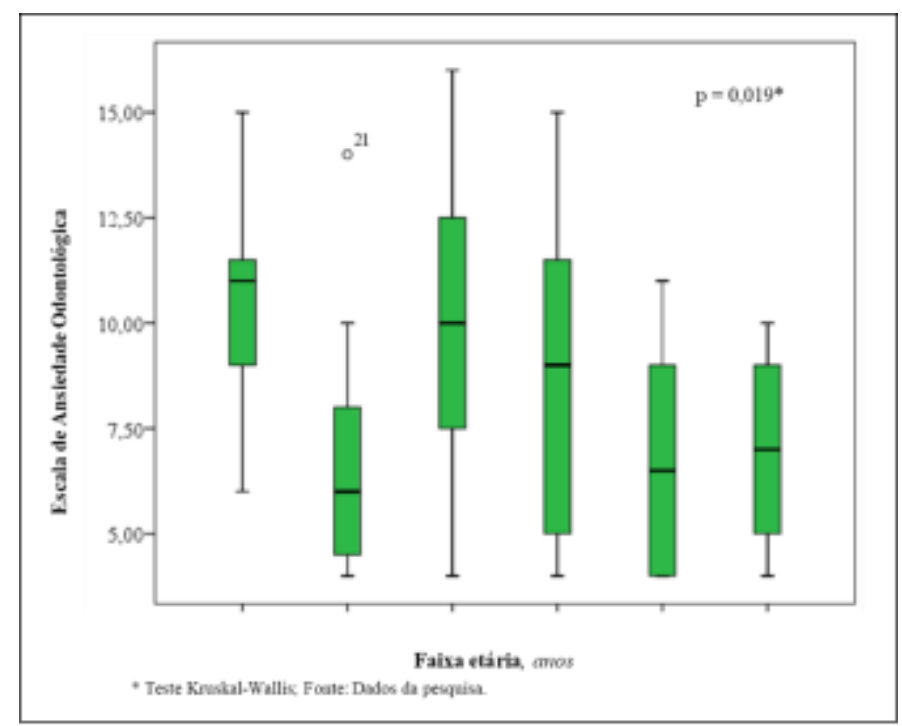

Fonte: Dados da pesquisa.

A distribuição dos níveis de ansiedade da amostra pelo grau de escolaridade não apresentou diferença estatística significante $(\mathrm{p}=0,414)$, conforme mostra o Gráfico 4. 
Gráfico 4. Distribuição da escala de ansiedade odontológica pela escolaridade da amostra.

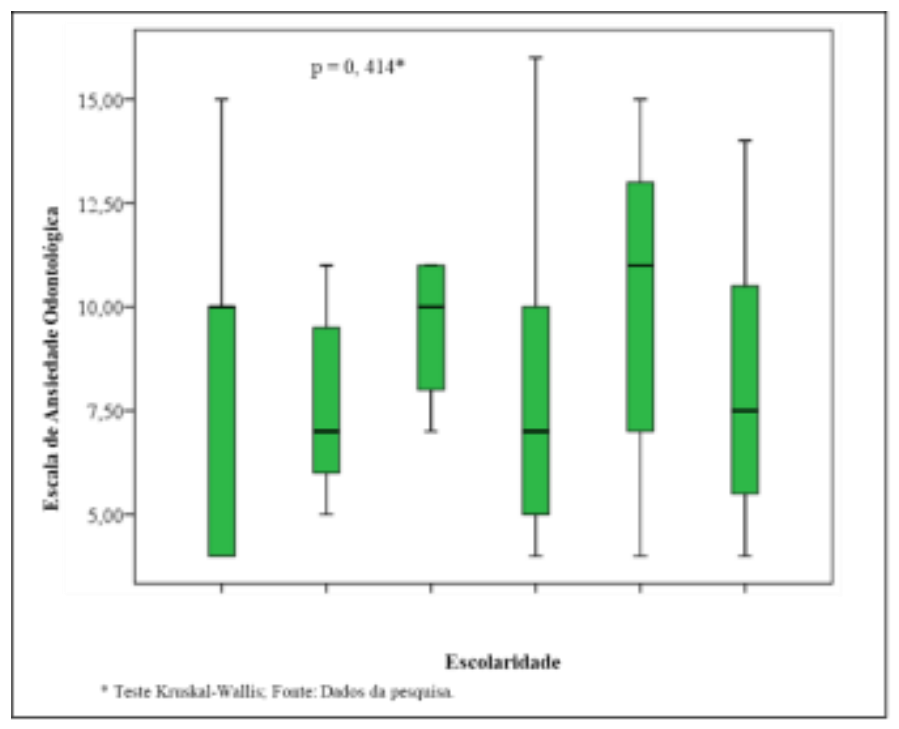

Fonte: Dados da pesquisa.

Ao interpretar o gráfico 5, nota-se que a distribuição da escala de ansiedade pela renda da amostra não obedece a nenhum padrão, o que implica na não correlação estatística entre as variáveis do estudo $(\mathrm{p}=0,228)$.

Gráfico 5. Distribuição da escala de ansiedade odontológica pela renda da amostra.

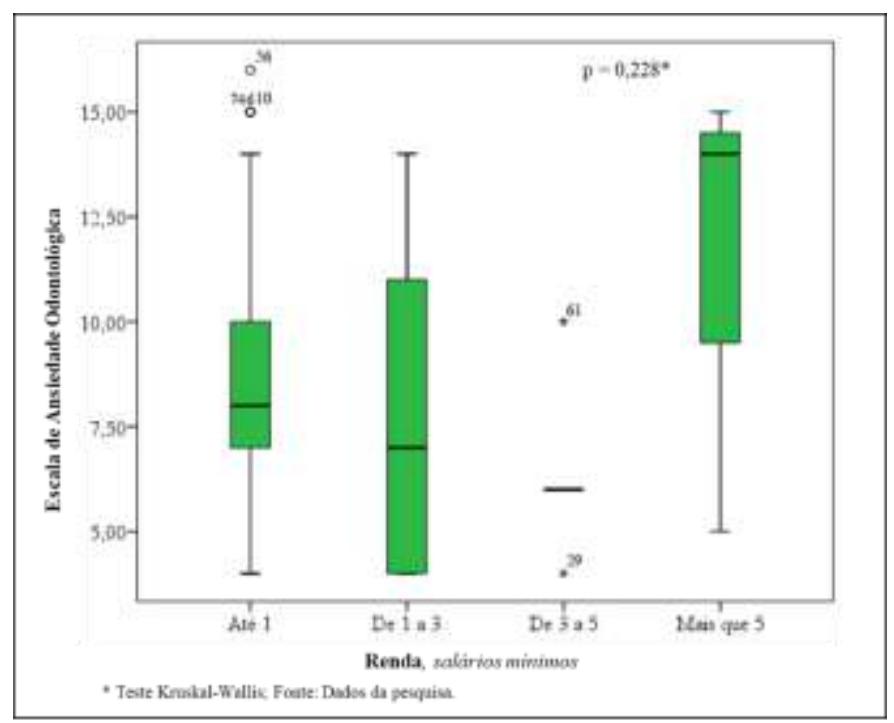

Fonte: Dados da pesquisa.

\section{Discussão}

A ansiedade dentária está intimamente ligada com o medo de sentir dor durante o tratamento odontológico, e ao se apresentar de forma frequente, pode desencadear um ciclo vicioso, que leva o paciente a buscar o tratamento odontológico somente quando sente dor (Appukuttan, 2016), o que vai de encontro aos resultados do nosso estudo.

Em relação à prevalência de ansiedade entre os sexos, o estudo de White, Giblin e Boyd (2017) relatam que as mulheres apresentam um maior nível de ansiedade dentária, o que contrapõe os achados da atual pesquisa, visto que não houve diferença estatística significante encontrada, assim como no estudo de Figueiredo, Coura, Oliveira, Penha e Medeiros (2020). 
Analisando os procedimentos que mais geram incômodo aos pacientes, a anestesia apresentou-se com maior prevalência na amostra estudada, revelando maior ansiedade a um procedimento simples. Em contrapartida, os estudos realizados por Figueiredo et al. (2020) e Teles, Cataldo, Schneider, Cardoso e Tannure (2016), trouxeram como predominante a alta rotação, o que mostra que mesmo com o avanço da tecnologia, não existem outros meios que exerçam a mesma função desses instrumentos e causem menos desconforto para substituí-los.

Levando em consideração o grau de escolaridade dos pacientes, a pesquisa não apresentou diferença estatística significante em relação à ansiedade ao tratamento odontológico, o que corrobora o estudo de Bottan, Pasini, Balestreri, Oliveira e Marín (2015), e se apresenta contrário ao estudo de Saeed, Hussein e Mahmood (2018), que alega que a baixa escolaridade está intimamente ligada à ansiedade, devido à falta de informação a respeito do tratamento odontológico.

O presente estudo, ao relacionar a faixa etária dos pacientes com a ansiedade odontológica, identificou uma tendência decrescente, uma vez que pessoas mais jovens possuíam maior ansiedade, enquanto maiores de 65 anos apresentaram menor ansiedade. Esses resultados respaldam os estudos de Caltabiano et al. (2018) e Giri, Pokharel, Gyawali e Bhattarai (2017), e contestam a pesquisa de Dantas, Marinho e Pinto (2019), que não obteve relevância significante. Já o trabalho de Pereira, Paula, Andrade e Castro (2017) demonstrou que o nível de ansiedade prevaleceu entre os 45 e 54 anos, indicando possíveis traumas.

Esse estudo apresentou uma diferença estatística não significante na relação da renda com a ansiedade dos pacientes e, no que se refere à frequência de comparecimento ao consultório odontológico, os resultados se mostraram relevantes nos pacientes que só se faziam presentes quando sentiam dor. Pereira et al. (2017) demonstraram resultados semelhantes aos apresentados, e Teles et al. (2016) corroboraram com o estudo no quesito renda, no entanto contrapuseram na frequência de comparecimento, onde a maioria dos pacientes procurou atendimento a cada 6 meses.

A Escala de Corah (1969) é tida como um excelente método avaliador de ansiedade dentária, e fazendo um comparativo com outros estudos, constata-se uma porcentagem considerável de pacientes com ansiedade leve. Souza, Lazzarin, Fideli, Tavares e Antonelli (2021), Rigo, Piano, Vieira, Sousa-Silva e Paranhos (2019), Saberi, Asli e Sharifiyan (2018), Lavaee, Khorshidi, Ghapanchi, Golkari e Kholousi (2017), obtiveram, respectivamente, as seguintes porcentagens: 36,2\%, 69,1\%, 41,5\% e $85 \%$, o que vai de encontro coma atual pesquisa, que resultou em $49 \%$ dos pacientes com ansiedade leve. Já segundo Figueiredo et al. (2020), o grau de ansiedade, no seu estudo, apresentou-se moderado (39,6\%).

Essa pesquisa a respeito da avaliação epidemiológica da ansiedade dos pacientes ao tratamento odontológico trouxe resultados claros. Entretanto, esperava-se um nível de ansiedade maior entre os pacientes, que pode ter sido reduzido devido a alguns fatores, como as habilidades clínicas e interpessoais eficazes dos estudantes, a ligação das pesquisadoras com a clínica escola e a apreensão pelo pouco tempo para responder o questionário, o que pode ter levado os pacientes a se sentirem envergonhados de relatar o seu verdadeiro nível de ansiedade.

Para obter sucesso no tratamento odontológico de um indivíduo com medo, o dentista deve adotar uma abordagem de tratamento apropriada adaptada às preocupações desse paciente. De acordo com Armfield e Heaton (2013), estratégias de redução da ansiedade relativamente simples também podem ser empregadas, como fornecer ao paciente senso de controle e previsibilidade em relação ao tratamento (Silva et al., 2021). Estar bem preparado para identificar com eficiência e oferecer um tratamento adequado baseado nos receios individuais de cada paciente é de extrema importância para a condução de um atendimento seguro sem maiores desgastes para ambos os envolvidos. O principal objetivo do cirurgião dentista deve ser acolher o paciente e aliviar seus medos e ansiedade para que eles se sintam motivados a longo prazo para futuras consultas odontológicas (Appukuttan, 2016; Fux-Noy et al., 2019).

As implicações derivadas da presente pesquisa mostram-se úteis, pois evidenciam o nível de ansiedade dos pacientes atendidos em uma clínica escola de odontologia, possibilitando identificar o que pode ser melhorado com o intuito de beneficiar a população atendida por esta instituição. No entanto, existem algumas limitações como a ausência de randomização na seleção 
dos pacientes, além dos nossos dados terem sido baseados em medidas auto-relatadas (questionário), que pode ser influenciado pelo viés do monométodo.

Dessa forma, outras pesquisas são recomendadas para permitir uma investigação mais aprofundada sobre o nível de ansiedade dos pacientes ao tratamento odontológico. Além disso, um estudo prospectivo apoiará dentistas e pacientes, com o potencial de abordar as desvantagens de um estudo transversal.

\section{Conclusão}

Os pacientes avaliados apresentaram, em média, um grau de ansiedade leve, sendo em sua maioria mulheres, com idade de 45 a 54 anos, renda de até 1 salário mínimo e $2^{\circ}$ grau completo. Os mesmos relataram comparecer ao dentista somente quando sentem dor, e afirmaram que a anestesia é o procedimento de maior incômodo. Se atentar à individualidade de cada paciente é primordial para um atendimento de sucesso, e entender as necessidades daqueles que cuidamos é o primeiro passo para que a ansiedade tenha uma incidência cada vez menor e a reabilitação oral seja realmente humanizada.

\section{Referências}

Appukuttan, D. (2016). Strategies to manage patients with dental anxiety and dental phobia: literature review. Clinical, Cosmetic and Investigational Dentistry, 35. https://doi.org/10.2147/ccide.s63626

Armfield, J. M., \& Heaton, L. J. (2013). Management of fear and anxiety in the dental clinic: a review. Australian dental journal, 58(4), 390-407; quiz 531. doi:10.1111/adj.12118

Bottan, E. R., Pasini, B., Balestreri, M., Oliveira, M. L. R. S. de, \& Marín, C. (2015). Relação entre ansiedade ao tratamento odontológico e fatores sociodemográficos: estudo com adultos em Santa Catarina (Brasil). https://secure.unisagrado.edu.br/static/biblioteca/salusvita/salusvita_v34_n1_2015_art_04.pdf

Caltabiano, M. L., Croker, F., Page, L., Sklavos, A., Spiteri, J., Hanrahan, L., \& Choi, R. (2018). Dental anxiety in patients attending a student dental clinic. BMC Oral Health, 18(1). https://doi.org/10.1186/s12903-018-0507-5

Corah, N. L. (1969). Development of a Dental Anxiety Scale. Journal of Dental Research, 48(4), 596. https://doi.org/10.1177/00220345690480041801.

Dantas, D., Marinho, V., \& Pinto, K. (2019). Prevalência de ansiedade frente à cirurgia oral: Um estudo com usuários da clínica escola de odontologia da Universidade de Gurupi. Amazônia Science and Health, 7(4), 69-77. https://doi.org/10.18606/2318-1419/amazonia.sci.health.v7n4p69-77

Figueiredo, C. H. M. da C., Coura, T. L. A. S., Oliveira, O. L. de, Penha, E. S. da, \& Medeiros, L. A. D. M. de. (2020). Nível de ansiedade dos pacientes submetidos ao atendimento odontológico. Archives of Health Investigation, 9(4). https://doi.org/10.21270/archi.v9i4.4724

Fux-Noy, A., Zohar, M., Herzog, K., Shmueli, A., Halperson, E., Moskovitz, M., \& Ram, D. (2019). The effect of the waiting room's environment on level of anxiety experienced by children prior to dental treatment: a case control study. BMC oral health, 19(1), 294. 10.1186/s12903-019-0995-y

Giri, J., Pokharel, P. R., Gyawali, R., \& Bhattarai, B. (2017). Translation and Validation of Modified Dental Anxiety Scale: The Nepali Version. International Scholarly Research Notices, 1-5. https://doi.org/10.1155/2017/5495643

Lavaee, F., Khorshidi, H., Ghapanchi, J., Golkari, A., \& Kholousi, S. (2017). The relation of preoperative stress and anxiety on patients' satisfaction after implant placement. Dental Research Journal, 14(5), 351. https://doi.org/10.4103/1735-3327.215968

Maulina, T., Djustiana, N., \& Shahib, M. N. (2017). The Effect of Music Intervention on Dental Anxiety During Dental Extraction Procedure. The Open Dentistry Journal, 11(1), 565-572. https://doi.org/10.2174/1874210601711010565

Pereira, C. M., Paula, P. H. F. de, Andrade, L. A. de, \& Castro, L. A. de. (2017). Avaliação do grau de ansiedade do paciente submetido a tratamento odontológico em uma Universidade de Goiânia/Goiás. Rev Ciências e Odontologia. 1(1),10-17. http://revistas.icesp.br/index.php/RCO/article/view/131/105.

Pereira, G. J. H. \& Queluz, D. P. (2000). Ansiedade dentária. Avaliação do perfil dos pacientes atendidos no setor público em Itatiba/SP. Rev Fac Odontol Bauru. 8(1),20-27.

Peronio, T. N., Silva, A. H. da, \& Dias, S. M. (2019). O medo frente ao tratamento odontológico no contexto do sistema único de saúde: uma revisão de literatura integrativa. Braz J Periodontol. 29(1),37-43. https://docplayer.com.br/134737817-O-medo-frente-ao-tratamento-odontologico-no-contexto-do-sistema-unicode-saude-uma-revisao-de-literatura-integrativa.html

Rigo, L., Piano, R., Vieira, W., Sousa-Silva, J., \& Paranhos, L. (2019). Evaluation of anxiety levels and their characteristics in dental care: Cross-sectional study. Indian Journal of Dental Research, 30(2), 300. https://doi.org/10.4103/ijdr.ijdr_325_18

Saberi, B. V., Asli, H. N., \& Sharifiyan, H. R. (2018). Evaluation of Dental Anxiety level and Related Factors in Patients Referred to Dental School. Jour Guilan Uni Med Sci 27 (106), 9-16. https://journal.gums.ac.ir/browse.php?a_id=1669\&sid=1\&slc_lang=en. 
Research, Society and Development, v. 10, n. 15, e347101522917, 2021

(CC BY 4.0) | ISSN 2525-3409 | DOI: http://dx.doi.org/10.33448/rsd-v10i15.22917

Saeed, D. N. A., Hussein, D. H. M., \& Mahmood, D. A. A. (2018). Prevalence of dental anxiety in relation to sociodemographic factors using two psychometric scales in Baghdad. Mustansiria Dental Journal, 14(1), 38. https://doi.org/10.32828/mdj.v14i1.753

Silva, A. F. M. da., Hasna, A. A., Bridi, E. C., Cunha, T. C. R. da., \& Silva, B. G. (2021). A relação entre ansiedade e o tratamento odontológico: pesquisa de campo. Research, Society and Development, 10(14), e142101421902. https://doi.org/10.33448/rsd-v10i14.21902

Silva, S. da. (2020). A musicoterapia como controle da ansiedade em pacientes adultos no consultório odontológico. Revista Cathedral, 2(1). http://cathedral.ojs.galoa.com.br/index.php/cathedral/article/view/59

Souza, J. G. M. V., Lazzarin, H. C., Fideli, G. C., Tavares, A. K., \& Antonelli, A. (2021). Avaliação do grau de ansiedade em pré-atendimento em clínica odontológica. Arquivos do Mudi, 25(2), 49-58. https://doi.org/10.4025/arqmudi.v25i2.58250

Stefano, R. de. (2019). Psychological Factors in Dental Patient Care: Odontophobia. Medicina (Kaunas, Lithuania), 55(10), 678. https://doi.org/10.3390/medicina55100678

Teles, L., Cataldo, D., Schneider, L. F. J., Cardoso, M. \& Tannure, P. N. (2016). Baixo nível de ansiedade dos pacientes atendidos no curso de odontologia de uma instituição de ensino superior. Rev. Odontol. Univ. Cid. São Paulo. 28(1), 24-9. https://docplayer.com.br/45623962-Luciano-teles-dionisia-cataldo-luisfelipe-jochims-schneider-mayra-cardoso-patricia-nivoloni-tannure-resumo.html

White, A. M., Giblin, L., \& Boyd, L. D. (2017). The Prevalence of Dental Anxiety in Dental Practice Settings. American Dental Hygienists' Association. February. 91(1), 30-34. https://jdh.adha.org/content/jdenthyg/91/1/30.full.pdf 